\title{
An Improved Impulse Noise Detection and Removal Method
}

\author{
Xie Dange-en, Zhang Zhili \\ (Network Centre of Xuchang University, Xuchang, China) \\ xde820@gmail.com, zzl@xcu.edu.cn
}

\begin{abstract}
This paper proposed an improved denoising method for removing the impulse noise. Firstly, analyze the each pixel of the corrupted image on eight directions, to determine whether the pixel is an impulse noise point. Then, create a noise identification matrix which has the same size of the original image, it can mark out the position of the noise point in the image. At last, with the guidance of the noise identification matrix, remove the impulse noise point one by one. The experiments are shown that the improved method has a larger PNSR value than the standard median filter(SM) algorithm, and retains a better details and sharper edges.
\end{abstract}

Keywords: Impulse Noise; Median Filter; Noise Removal; Noise Detection

\section{Introduction}

During the acquisition or transmission, digital images are often corrupted by impulse noise [1], which is independent of image content. An important type of impulse noise is salt-and-pepper noise, which is usually found in imaging with quick transients such as faulty switching, decoding errors, or transmitting images over noisy digital channels. When images are corrupted by salt-and-pepper noise, only parts of pixels are changed, and the noisy pixels like white and black dots sprinkled on the images. Most classical image denoising methods are low pass filtering method, in which the median filtering method is widely used. Since the method for removal of impulse noise has good performance, it was first used for removal of impulse noise [2].However, in the SM algorithm, noise removal based on reducing the image resolution, so the result image is fuzzy. With the increase of the filter window, the result of image blurring increases.

In recent years, appeared a variety of improved algorithm based on median filtering, such as adaptive median filter [3-5], weighted median filtering algorithm [6], this algorithm improves the performance of median filter in respect to make beneficial exploration, but each has its limitations, mainly in the processing of all of the pixels in filtering noise, but also change the noise pixel gray value, thereby causing image blur. Obviously, the ideal filtering algorithm should discriminate between noise and signal, only the noise pixel median instead, while keeping the signal pixel gray value unchanged.

On this basis, this paper puts forward a noise detection of impulse noise removal method. This method by setting the threshold to mark out the possible noise points, and then according to the relative information of adjacent pixels may be noise points further judgment, to accurately determine the noise points. This can reduce image blur and improve image quality. The experimental results show that, the algorithm can effectively remove the noise, while preserving the 
details of the image, is an effective image noise filtering algorithm.

\section{Algorithm Method}

The improved method contains two levels. First, identify the noise points. Second, replace them with the pixel data, and then to remove them.

\subsection{Impulse Noise detection}

Impulse noise detection is a very important part in the median filtering algorithm; it determines the correct classification of the image pixels. Impulse noise from images accurately detected can promote the image quality after median filtering. The impulse noise is due to some reasons in the image to produce a number of gray values are very small (almost black) or gray value large (close to white) pollution, is concentrated in the extreme 0 or 255 near the point of extreme value.

Set pulse noise intensity range is $[0, \omega]$ and [255- $\omega, 255]$, if the image of a pixel gray value in the two ranges, the pixel as suspicious noise points, and for signaling point. But not all suspicious noise points are pulse noise, it may also be a narrow edge of image, thin and flat gray area, so this is just a preliminary determination, by simply based on impulse noise point of some characteristic to judge is not the true nature of the pixels of the display.

\subsection{Noise Identification Matrix}

In order to identify the pulse noise point location, establishment a Boolean matrix which has the same size as the noisy image dimension, called noise identification matrix, denoted as $N$. Matrix of each element to be processed with the noisy image in which each pixel corresponding to, denoted by $N_{(i, j)}$, then

$$
N=\left\{N_{(i, j)} \in\{0,1\}, 1 \leq i \leq h, 1 \leq j \leq w\right\}
$$

Here, $\mathrm{H}$ and $\mathrm{W}$ denote the height and the width of the image respectively. When $N_{(i, j)}$ is equal to 0 , it indicates that there is an impulse noise point, 1 said the original image pixel. Firstly, we initialize $\mathrm{N}$ is full of 1 , and then modify the data as 0 selectively during the noise detection process.

\subsection{Determine the Noise Points}

A large amount of data shows that the image adjacent pixels have the gray value are closely linked, that is the spatial adjacent pixel gray value is very close.

Here, we divide the 25 pixels in a $5 \times$ 5 window into eight groups of different direction of the window to be detected. Figure 1 shows the 8 windows, the center pixel is the suspicious noise pint. The groups can detect the noise point on every $\pi / 8$ direction between $[0, \pi]$. In each $5 \times$ 5 window, we use the suspicious noise gray value multiplied by the number of other pixels in the window on the direction firstly. Secondly, make the differential with the gray values of the pixels within the window, thus obtained 8 groups representing the eight windows the differential gray value. Finally, make the further analyze of the differential gray values in order to accurately locate the position of impulse noise points.

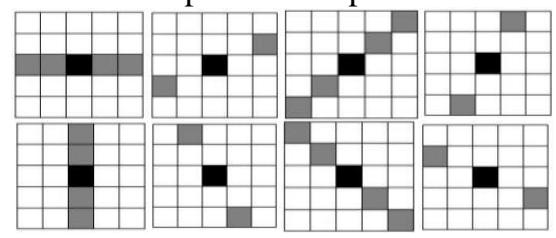

Fig. 1 Noise Detect windows on eight directions

Let $P$ be the current suspicious noise points in the center of the $5 \times 5$ window, and it's intensity value is $f(P)$, $f_{N}(P)$ stands for the neighbor pixel value of $P$. Let $d_{i}$ be the differential gray value between $P$ and it's neighbor pixels on each direction, then 


$$
\begin{aligned}
d_{i} & =n \cdot f(P)-\sum f_{N}(P), \\
i & =\{0 \cdots 7\}, \\
n & =\{2,4\} \\
d & =\min \left\{\left|d_{0}\right|,\left|d_{1}\right|, \cdots,\left|d_{7}\right|\right\}
\end{aligned}
$$

Let $d$ is the minimal absolutely value of $d_{i}$, then we can determine whether the pixel $P$ is an impulse noise point based on the value of $d$. If the current center pixel $P$ is a flat area point, most pixel value of its 24 adjacent pixel in the $5 \times 5$ window will be very close to it, so the value of $d$ is small. If $P$ is the image edge point, then in the eight window, at least one window's pixel gray value is close to it, so the value of $d$ is also small. If $P$ is the noise point, a large difference is existed between the adjacent pixels and itself. So each $d_{i}$ is large, and $d$ is large too. So here we set a threshold called $\tau$, if $d$ is larger than $\tau$, the current center pixel $P$ is a noise point. Otherwise, it is an unpolluted signal point. The choice of the threshold value $\tau$ is determined by comparing the most appropriate value in the experiment. At last, when $P$ is determined a noise pint, set the corresponding element's value of the Matrix $N$ is 0 , others are set to be 1 , that is

$$
N_{(i, j)}=\left\{\begin{array}{cc}
0, & d>\tau \\
1, & \text { otherwise }
\end{array}\right.
$$

where ${ }_{N_{(i, j)}}$ equal to 0 or 1 representing whether the current pixel is a noise point in the original image.

\subsection{Impulse Noise Removal}

When the noise identification matrix $\mathrm{N}$ is defined, we can do the next step, which is the impulse noise removal. From the guidelines of the logical matrix $N$, we just filter out the noise points by the SM algorithm one by one, but the signal pixels are not changed. Compared with the SM algorithm, our improved method contains two levels' classification. The meth- od can avoid the erroneous classification efficiently and has a better effect on denoising and keeping the image details. That means our improved method can find a good balance on protecting image details and impulse noise removal.

\section{Experiment Result}

All the experiments are completed under the Win7 32 bits OS by MATLAB (R2009a) programming language, and the $\mathrm{PC}$ is equipped with Intel Core2 Duo $2.53 \mathrm{GHz}$ CPU and 2GB RAM memory. A $256 \times 2568$-bit gray-level homogenous image "lena" that has many features is selected in our experiments. The performance of the results is quantified by the peak signal-to-noise ratio (PSNR) [7].

Table 1 Comparison of restoration results in PSNR (dB) for image corrupted with impulse noise

\begin{tabular}{|c|c|c|c|c|c|}
\hline \multirow{2}{*}{ Method } & \multicolumn{5}{|c|}{ Noise Level } \\
\cline { 2 - 6 } & $10 \%$ & $20 \%$ & $30 \%$ & $40 \%$ & $50 \%$ \\
\hline SM(3×3) & 27.49 & 25.75 & 21.92 & 18.30 & 14.79 \\
\hline SM $(5 \times 5)$ & 26.46 & 25.07 & 23.54 & 22.39 & 20.22 \\
\hline SM(7×7) & 25.03 & 23.50 & 22.26 & 21.45 & 20.35 \\
\hline Ours & 29.39 & 26.83 & 25.10 & 23.78 & 21.96 \\
\hline
\end{tabular}

Table 1 lists the PSNR values of different methods in different noise level. In the experiment, the parameters $\omega$ and $\tau$ values were 12 and 15 . Compare the data in the table, we can find our method gets a larger PSNR value. Figure 2 shows the PSNR values of denoising result for different noise levels by some methods based on SM algorithm in different filter window size $(3 \times 3,5 \times 5,7 \times 7)$ and ours. The data is derived from Table 1 .

In order to compare subjective image qualities, the restored images by different methods for images corrupted with $40 \%$ impulse noise are presented in Figure 3. It is easy to see that the restored images by our method are clearer and sharper than the SM method. 


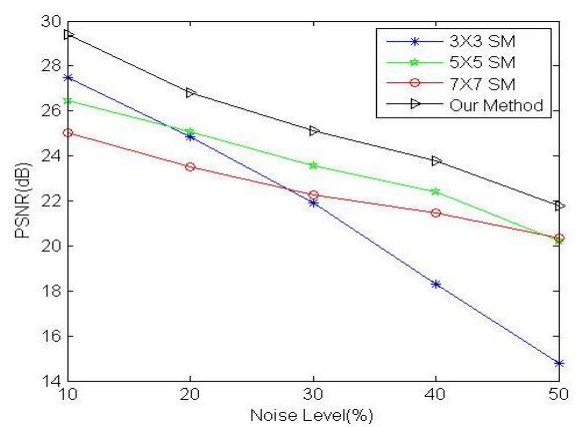

Fig. 2 PSNR values for different methods

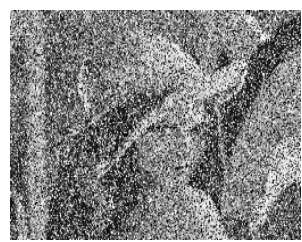

(a)

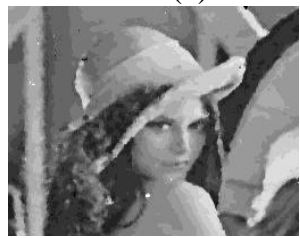

(c)

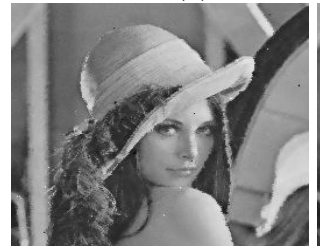

(e)

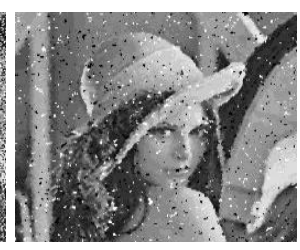

(b)

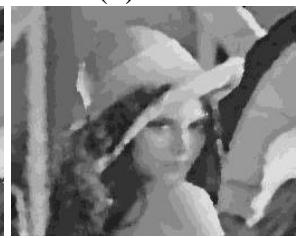

(d)

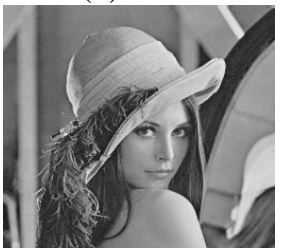

(f)
Fig. 3: Results of different methods in restoring 40\% corrupted image "lena": (a) the noise image $(9.44 \mathrm{~dB}),(\mathrm{b})$ the $3 \times$ $3 \mathrm{SM}$ filter $(18.30 \mathrm{~dB}),(\mathrm{c})$ the $5 \times 5$ SM filter $(22.39 \mathrm{~dB}),(\mathrm{d})$ the $7 \times 7 \mathrm{SM}$ filter $(21.45 \mathrm{~dB})$, (e) our method $(23.78 \mathrm{~dB})$, (f) the original image.

\section{Summary}

Basically, our method can be regarded as an improved algorithm for the SM algorithm. Because the improved method just filtering the noise points, the normal signal value is unchanged, the results are sharper and clearer than the SM algorithm.

\section{Acknowledgment}

This work is supported by the NSF (No. 2009A520024) of Henan Province and the fund of the science \& technique bureau of Xuchang city (No.1103018, No.1101031).

\section{References}

[1] Yujin Zhang, Image Engineering: Image Processing (Second Edition) [M], Tsinghua University Press, Beijing, China, 2006.

[2] Pratt W K. Median filtering. Image Processing Technique report, Univ. of Southern California, Los Angeles, 1975, Sept.

[3] Hwang H , Haddad R A. Adaptive median filters: new algorithms and results [J]. IEEE Trans. Image Processing, 1995, 4: 499-502.

[4] Chunyu Ning, Chunhua Zhao, Removing impulse noise in medical images using adaptive median filtering algorithm[J], Computer Engineering and Applications(in Chinese), 2012, 48(24):153-156.

[5] Wei Wang, Jinghuai Gao, Random seismic noise suppression via structure-adaptive median filter[J], Chinese Journal of Geophysics(in Chinese), 2012,55(5):1732-1741.

[6] Yu Zhang, Xiqin Wang,Adaptive center weighted modified trimmed mean filter[J]. Journal Of Tsinghua University (Science \& Technology), 1999, Vol. 39(9):

[7] Gonzalez R C and Woods R E. Digital image processing [M]. AddisonWesley, Boston, 2002. 